\section{A GROUNDED THEORY EM UM CONTEXTO MULTIDISCIPLINAR: CARACTERISTICAS E CONTRIBUIÇÕES NO CONTEXTO DA ENGENHARIA E GESTÃO DO CONHECIMENTO}

FRANCISCO, Thiago Henrique Almino. - Doutor, pelo Programa de Pós-Graduação em Engenharia e Gestão do Conhecimento da Universidade Federal de Santa Catarina (EGC/UFSC). Mestre, pelo Program de Pós-Graduação em Administração Universitária da Universidade Federal de Santa Catarina (PPGAU/ UFSC); NAKAYAMA, Marina Keiko. - Mestrado e Doutorado em administração na UFRGS; VEFAGO Yuri Borba*. - Graduado, pelo curso de Administração de Empresas da Universidade do Extremo Sul Catarinense (UNESC). *Autor para correspondência e-mail: yurivefago@gmail.com

Recebido em: 28/08/2017 Aprovação final em: 21/11/2017

Resumo

O artigo contextualiza a Grounded Theory como elemento e estratégia metodológica em um contexto multidisciplinar, tendo como objeto de investigação as teses e dissertações de um programa de pós-graduação que se caracteriza dessa forma. O referencial teórico apresenta uma visão geral da investigação qualitativa e da Grounded Theory como estratégia metodológica, posicionando-a no contexto interpretativista e qualitativo. A partir de uma revisão integrativa de literatura, o artigo analisa os trabalhos que foram desenvolvidos, a partir da utilização da Grounded Theory no contexto da Engenharia e Gestão do Conhecimento. Os resultados demonstram uma importante evolução dos estudos qualitativos no programa a partir da adoção da Grounded Theory como elemento de pesquisa, permitindo que novos estudos se desenvolvam e colaborando com a consolidação de linhas que se posicionam sob o viés qualitativo, tendo no conhecimento o principal objeto de estudo.

Palavras-chave: Grounded Theory; Gestão do Conhecimento; Multidisciplinaridade.

The Grounded THEORY IN A MULTIDISCIPLINARY BACKGROUND: FEATURES AND CONTRIBUTIONS IN THE CONTEXT OF ENGINEERING AND KNOWLEDGE MANAGEMENT

\section{Abstrac}

The article contextualizes the Grounded Theory as an element and methodological strategy in a multidisciplinary context, having as object of investigation the theses and dissertations of a postgraduate program that is characterized in this way. The theoretical framework presents an overview of qualitative research and Grounded Theory as a methodological strategy, placing it in an interpretative and qualitative context. From an integrative literature review, the article analyzes the work that was developed, based on the use of Grounded Theory in the context of Engineering and Knowledge Management. The results demonstrate an important evolution of the qualitative studies in the program since the adoption of Grounded Theory as research element, allowing the development of new studies and collaborating with the consolidation of lines that are positioned under the qualitative bias, having knowledge as the main object of study.

KEYwORDS: Grounded Theory; Knowledge Management; Multidisciplinary.
VoL. 21, N.1, 2018
INTRODUÇã̃o

A Grounded Theory é considerado por alguns autores como um método de pesquisa, bem como um metodologia em si mesma (STRAUSS; CORBIN, 1998), um estilo ou abordagem (LOCKE, 2001) ou ainda pode ser entendida como uma estratégia de pesquisa (WELLS, 1995). Segundo Strauss e Corbin (1998), a Grounded Theory oferece uma estrutura metodológica às vezes ausente em outras abordagens qualitativas, sem sacrificar a flexibilidade ou rigor a pesquisa. Esta teoria está fundamentada em dados desenvolvidos na década de 1960 por dois sociólogos, Barney Glaser e Anselm Strauss, publicadas no livro The Discovery of Grounded Theory.

Naquela época, a introdução da teoria Grounded Theory representou uma reação as metodologias ja existentes, e se estabeleceu como uma alternativa à hegemonia da lógica hipotético-dedutiva dos trabalhos de orientação positivista nos estudos sociológicos (CHARMAZ, 2006). Glaser e Strauss (1967) estavam decididos em desenvolver uma metodologia, ou um estilo de se faze pesquisa, que fosse capaz de preencher o espaço existente entre o que eles chamaram de "pesquisas empíricas teoricamente desinteressadas" e de "teorias empiricamente desinteressadas", que predominavam nas ciências sociais até então. Para os autores as teorias geradas segundo a lógica positivista científica da época, apesar de serem formuladas de maneira muito sistematizada, ou justamente por esse excesso de sistematização, se revelavam muito abstratas e tinham muito mais a ver com os (pré) conceitos teóricos prévios dos pesquisadores, do que propriamente com a "realidade empírica" (PINHO \& SANTOS, 2012)

Para Pinho e Santos (2012), este tipo de teoria se encaixa bem porque é construída com conceitos e categorias que surgem a partir dos termos que os próprios agentes sociais usam para interpretar e organizar o seu mundo. Uma "boa" teoria substantiva deve ser útil no dia a dia não apenas para os sociólogos, mas para os leigos. É necessário que seja compreensível, relevante para a prática e aplicável em diferentes situações e condições à luz da realidade cotidiana das pessoas. Conseguir isso não é, de fato uma tarefa fácil. (LOCKE, 2001).

Sob esse pano de fundo, o trabalho busca elucidar as características e contribuições dos estudos utilizaram a Grounded Theory, sob uma perspectiva qualitativa, em um programa de pósgraduação multidisciplinar

A proposta deste trabalho está ancorada em um estudo bibliográfico que guarda as características de uma revisão integrativa de literatura, considerando a proposta definida por Whittemore e Knafl (2005), como um método que contribui com a organização de estudos empíricos para uma determinada finalidade de pesquisa. Para tanto, resta definir com clareza a finalidade do estudo e suas intenções propositivas, as quais permitem posicionar a investigação em um determinado plano epistemológico. É nesse sentido que a pesquisa se estrutura na perspectiva do paradigma interpretativista, de viés fenomenológico, dada as características análogas ao que definem Burrel e Morgan (1979).

Sob uma abordagem qualitativa, o estudo busca efetuar uma leitura de um determinado contexto a partir do que consideram Taylor e Bogdan (1984), tendo em vista a busca por evidencias de fenômenos complexos, mas que possam se apresentar livre das concepções herméticas que traços característicos de algumas áreas do conhecimento. $\mathrm{O}$ caso aqui é buscar um entendimento de um fenômeno livre de concepções finitas, por meio do levantamento de estudos que tratam de construtos que interessam ao investigador e, por consequência, a comunidade que estuda o fenômeno em discussão neste artigo.

Nesse sentido, a proposta do material é desenvolver um estudo que contemple a revisão dos materiais publicados na Base de Teses e Dissertações do Programa de Pós-Graduação em Engenharia e Gestão do Conhecimento da Universidade Federal de Santa Catarina, com o intuito de e os resultados dos estudos que utilizaram a Grounded Theory com estratégia metodológica 
da pesquisa. Para tanto, dois critérios de inclusão foram escolhidos para contribuir com a escolha dos estudos, os quais seguem em destaque:

- Estudos que se configurem como Teses ou Dissertações, defendidos no programa de Pós-Graduação em Engenharia e Gestão do Conhecimento da Universidade Federal de Santa Catarina;

-Estudos que citem no título e (ou) no resumo o uso da Grounded Theory como estratégia da pesquisa;

Ademais, é valido ressaltar que o estudo não se propõe a avaliar a teoria substantiva produzida pelos trabalhos, caso isso tenha ocorrido.

A pesquisa qualitativa: uma visão geral do método em suas principais caracteristicas

O campo das ciências humanas e sociais oferece duas visões metodológicas distintas à realização da pesquisa científica. Uma delas adota os métodos quantitativos de pesquisa, o qual enfoca o comportamento humano como resultado de forças, fatores, estruturas internas e externas que atuam sobre as pessoas. Esta visão é chamada de Positivista e segundo os defensores desta visão, esta pesquisa pode ser estuda pelo método experimental por levantamentos amostrais.

Moreira (2012) destaca que o positivismo teve sua origem em Auguste Comte e John Stuart Mill. Suas obras advogam que as ciências humanas e sociais realizam pesquisas através das ciências físicas. Para Comte (1978), "o estado positivo caracteriza-se, pela subordinação da imaginação e da argumentação à observação. Cada proposição enunciada de maneira positiva deve corresponder a um fato, seja particular, seja universal.

Já na visão qualitativa os estudos são pautados na interpretação do mundo real, preocupando-se com o caráter hermenêutico na tarefa de pesquisar sobre a experiência vivida dos seres humanos. Esta abordagem tem suas raízes no final do século XIX. Foi na área das ciências sociais que primeiro se questionou a adequação do modelo vigente de ciência aos propósitos de estudar o ser humano, sua cultura, sua vida social.

Para Godoy (1995) os estudos de pesquisa qualitativa apresentam diferença entre si quanto aos método, os objetivos e a forma. $\mathrm{O}$ autor enumera em conjunto de características que identificam a pesquisa qualitativa a saber: a) o ambiente natura como fonte direta de dados e o pesquisador como instrumento fundamental; b) o caráter descritivo; o significado que as pessoas dão as coisas e a sua vida como preocupação do investigador; e o c) enfoque indutivo. Para o autor a expressão pesquisa qualitativa assume diferentes significados no campo das ciências sociais e compreendem um conjunto de técnicas interpretativas que diferem entre si, e que visam descrever e a decodificar os componentes de um sistema completo de significados.

Maanen (1979) completa dizendo que estes estudos são feitos em sua maioria no local de origem dos dados e possibilita o pesquisador empregar a lógica do empirismo cientifico (adequada aos fenômenos claramente definidos) porém é bem mais apropriado empregar a perspectiva da análise fenomenológica quando se tratar de fenômenos singulares e dotados de certo grau de ambiguidades.

Quanto as formas da pesquisa qualitativa apontamse pelo menos tres diferentes possibilidad a pesquisa documental, o estudo de caso e a etnografia. A pesquisa documental pelo exame de materiais que ainda não receberam um tratamento analítico ou que podem ser reexaminados com Já o estudo de caso visa ao exame detalhado de um ambiente, de um sujeito ou de uma situação em particular. Este tipo de estudo tem se tornado a modalidade preferida daqueles que procuram saber como e por que certos fenômenos acontecem, e permitem ainda analisar eventos sobre os quais a possibilidade de controle é reduzida ou quando os fenômenos analisados são atuais e só fazem sentido dentro de um específico contexto. Entretanto o estudo da etnografia tem se destacado como um dos métodos mais importantes por envolver o pesquisador por longo período de estudo, onde o mesmo adotará o metodo de observação, contato direto e participação em atividades. do fenômeno. O que importa, nestes estudos, não é forma de que os fatos se revestem, mas sim, o seu sentido. (GODOY, 1995)

A grounded theory: a caracterização de um método complexo sob o viés interpretativista

$\mathrm{Na}$ condição atual da ciência, a produção do conhecimento perpassa o entendimento simplório da conjunção de termos que estão atrelados a um determinado objeto ou fenômeno em investigação. $\mathrm{O}$ processo deve considerar uma visão complexa de mundo e o entendimento dos paradigmas que envolvem essa construção, os quais suportam os pressupostos que configuram uma determinada natureza da sociedade. É nessa visão que Burrel e Morgan (1979) sustentam a possibilidade da construção do conhecimento científico de forma multiparadigmática, enfatizado a segmentação de visões a partir de dimensões da teoria social.

Em especifico, a visão de mundo interpretativista carrega consigo desafios que estão materializados no campo da ciência. Para Silveira (1997), o campo da ciência, sob qualquer viés, deve buscar a ruptura com paradigmas conservadores e avançar para padrões que permitam e promovam o avanço significativo do processo científico, determinando a (re) construção dos padrões epistemológicos que orientam o desenvolvimento de uma nova proposição para a produção de conhecimento.

Para Rosso, Bandeira e Costa (2002), o desenvolvimento de novos padrões propositivos para a ciência deve permitir a reinterpretação do mundo, considerando-o algo interpretável na visão do sujeito, desprovido de uma visão hermética de um determinado fenômeno. Isso reconfigura a construção do conhecimento e promove uma espécie de "revolução" na proposta científica, enfatizando a participação do sujeito nas construções sociais que interpretam um determinado domínio de conhecimento.

É nessa perspectiva que se posiciona a Grounded Theory na visão proposta por Strauss e Corbin
(1990), apresentando-a como um método genérico que transitou em diversos paradigmas até se constituir em uma estratégia metodológica de pesquisa focada no desenvolvimento de estudos sob a ótica interpretativa e construtivista. Na visão dos autores, a Grounded Theory preconiza um envolvimento prático com o fenômeno em estudo, permitindo que haja a integração entre o fenômeno em estudo e o sujeito, de modo que ambos possam fazer parte da natureza social em estudo. Como a Grounded Theory é um método descritivo e explicativo, ela deve, necessariamente, permitir que o objeto seja compreendido como um processo social básico, elevado, a partir da metáfora do interacionismo simbólico, a uma condição de fenômeno conjuntural complexo, que utiliza várias fontes para que seu padrão seja compreendido e reconfigurado.

Dey (1999) apresenta a Grounded Theory como um método fundamentado em dados que é caracterizado de distintas formas ao longo de sua evolução, sempre tendo como objetivo a promoção de um avanço profundo em uma determinada área de conhecimento por meio do estudo de um fenômeno, objeto ou processo social. A construção das teorias substantivas, na visão do autor, foi preponderante para a consolidação da proposta metodológica em questão, mesmo nos momentos de críticas em função de sua condição multiparadigmática. Mesmo assim, em meio a críticas de diversos segmentos da comunidade científica, a Grounded Theory se posiciona como um elemento profícuo na produção de conhecimento científico sob o viés qualitativointerpretativista, de modo a conduzir a construção de teorias aplicadas a um determinado contexto.

Assim caracterizado, o método, também considerado por Strauss e Corbin (2008) como uma estratégia metodológica, se estabelece como a base de uma proposição de avanços para a comunidade científica em função da possibilidade de promover teorias substantivas que sustentam um determinado conjunto de suposições, que sob a égide do viés qualitativo podem fortalecer um determinado campo de investigação. A contribuição de Mills, 
Bonner e Francis (2006) permitem uma visão condições.

melhor deste entendimento, ao destacarem que a:

Grounded Theory has proved an enduringly popular choice of methodology for nurse researchers since its development in the 1960 s, with more than 3,650 journal articles published, both on the ethodology itself and reporting research outcomes. (MILLS, BONNER E FRANCIS, 2006, p. 2)

$\mathrm{Na}$ perspectiva interpretativista, destarte, a Grounded Theory carrega a essência da proposta de Strauss e Corbin (2008), que sustentou a construção do método sob um olhar qualitativo, e se caracteriza como um método que utiliza critérios e paradigmas variados para o entendimento de um determinado fenômeno, assumindo uma estrutura que deve ser avaliada com muita atenção em função das demandas que o objeto de estudo impõe ao método Dessa forma, o método, também considerado uma estratégia metodológica, aponta para o uso de instrumentos específicos e fundamentados nos seguintes elementos, que são destacados por Corbin e Strauss (1990):

-O envolvimento "prático" do pesquisador com um determinado processo social básico a partir das atividades de descricão e explicação do fenômeno;

- A compreensão das condições específicas do objeto de estudo pelo uso de diferentes estratégia de pesquisa;

-O entendimento de um princípio que reque o entendimento do pesquisador sobre a constante mudança do fenômeno por isso ele deve rejeitar o determinismo.

E sob estas condições que a Grounded Therory carrega seus fundamentos para a produção e o desenvolvimento de teorias substantivas, que em sua grande maioria são elementos que se aplicam a condições complexas e fenômenos específicos, que carregam conotações simbólicas para uma determinada comunidade que investiga e sistematiza os elementos que emergem dessa
Os estudos multidisciplinares: uma

Em conjunto com a interdisciplinaridade, a multidisciplinaridade discutida por Sommerman (2006) se apresenta como um movimento dinâmico que é contra a fragmentação disciplinar que envolve as disciplinas que permitem as mais diversas visões de mundo. Ao buscar compreender essa dinâmica, busca-se também a compreensão de um dialogo metodológico que é envolto por uma série de epistemologias, configurando um cenário, já apresentado por Creswell e Plano Clark (2013), de "metamorfose" científica, fortalecendo conceitos e métodos que buscam a construção constante de uma nova epistemologia.

Desde uma evolução filosófica até a construção técnica que integra visões de mundo, o conceito de multidisciplinar idade, bem como os demais que o acompanham, buscou, ao longo da evolução, uma ruptura epistemológica que permitisse a transposicão do conhecimento para as diversas classes "sócio-intelectuais", impedindo que o reducionismo e a fragmentação e fortalecendo a construção do conhecimento de forma reflexiva e orientado pela dialética.

Fava (2014), ao discutir a nova educação para o século XXI, destaca que a fragmentação disciplinar mpede que o conhecimento se democratize a partir das diversas visões, fazendo com que uma visão de mundo possa se reduzir a uma definição ou a um posicionamento de apenas uma disciplina. Cardoso et al (2008), também destacam que esse movimento é algo que deve ser revisto no contexto de uma nova sociedade, que busca o fortalecimento da consciência científica que possa ampliar uma visão social, evitando observar uma tradição positivista que historicamente foi observada ao longo do processo educacional.

Nesse diálogo sistemático em busca da transposição dos saberes, Brunn et al (2005), também destaca que o entendimento da inter e da multidisciplinaridade, além de busca rupturas epistemológicas, também se apoia na construção do conhecimento por um processo dialético, contrario a fragmentação disciplinar. Além de observar um aprofundamento para fora das correntes racionalistas, o conceito defendido por Sommerman (2006) buscava fortalecer os aspectos concretos do iluminismo, permitindo que a ciência moderna estivesse constantemente dialogando com os saberes.

Voltando para a metáfora educacional, tanto a inter quanto a multidisciplinaridade como prática ensejam que os currículos possam ser reajustados de modo a entender a dinâmica complexa do mundo, fortalecendo uma visão triangular dos fenômenos. É por influência da tecnologia que esse movimento ganha corpo, principalmente pela imensidão de novas informações que se criam e que determinam o incremento de novos conteúdos que permitem a visão de um fenômeno complexo por diversas óticas.

A visão complexa do conhecimento, nesse contexto, se constitui pelo fato de que a visão ortodoxa do conhecimento é substituída por uma concepção livre, porém orientada por um objetivo concreto que é relacionado com a construção do conhecimento. Essa visão, a qual corre numa perspectiva objetiva-subjetiva, faz com que a visão de mundo possa se fortalecer ao longo de um determinado fenômeno, contando com a colaboração das estruturas cognitivas das diversas disciplinas para o estabelecimento de uma nova definição, a qual conta com a colaboração da inter e da multidisciplinaridade.

Apresentação dos resultados da pesquisa: os

Pela dinâmica da proposta educacional brasileir a condição multidisciplinar vem ganhando um notório espaço na produção de conhecimento no cenário nacional, sobretudo pelo incentivo das estruturas sociais que determinam os investimentos em inovação, ciência e tecnologia. O Plano Nacional de Pós-Graduação 2010-2015 (CAPES 2010) determina que esses conceitos sejam desenvolvidos em uma condição social holística, fortalecendo o desenvolvimento de ações que possam sensibilizar a sociedade em função da dinâmica que demanda ações de inovação.

Nesse contexto, surge e se posiciona o programa multidisciplinar desenvolvido pela Universidade Federal de Santa Catarina, que se constituiu como um marco inovador no cenário da Pós-Graduação Stricto-Sensu no Brasil. A Pós-Graduação em Engenharia e Gestão do Conhecimento, cuja implantação ocorreu no ano de 2004 e se consolidou em uma perspectiva que envolve três áreas de concentração, considerando a Engenharia do Conhecimento, a Gestão do Conhecimento e as Mídias do Conhecimento, articula propostas que possam exploram o conhecimento como o principal objeto de estudo e sustenta as visões de mundo multiparadigmáticas em sua estrutura curricular. No intuito de observar o conhecimento como o principal elemento que gera valor para os negócios e para a atual sociedade do conhecimento, o programa atua de modo a promover a integração entre a academia e o setor produtivo, de maneira que a inovação e o conhecimento tornem-se proposições de valor para a sociedade.

Especificamente na área da gestão do conhecimento, considerando a linha de pesquisa em "Teoria e Prática em Gestão do Conhecimento", a proposta do programa de estudar o conhecimento como objeto de estudo é desenvolvido nos níveis de mestrado e doutorado, utilizando para isso os preceitos metodológicos defendidos pelo programa. Nesse sentido, um dos grupos de pesquisa ligados ao Departamento de Engenharia e Gestão do Conhecimento, vem desenvolvendo atividades que envolvem 0 estudo do objeto defendido pelo EGC a Grounded Theory.

O Núcleo de Estudos em Observação, Pessoas e Aprendizagem (NEOGAP), desde a sua fundação ja desenvolveu diversos trabalhos que colacionam a Grounded Theory e o conhecimento na perspectiva da gestão do conhecimento, os quais passam a ser caracterizados a seguir. 


\section{Os estudos em análise}

Os estudos escolhidos para a análise são referências em função de se constituírem em um conjunto de materiais que foram desenvolvidos sob a orientação de princípios estabelecidos em um dos grupos de pesquisa que estão alocados no Programa de Pós-Graduação em Engenharia e Gestão do Conhecimento da Universidade Federal de Santa Catarina. A partir das atividades do Núcleo de Observação em Gestão, Aprendizagem e Pessoas (NEOGAP), é possível perceber o início do uso da Grounded Theory como um elemento metodológico aplicado ao estudo de fenômenos complexos no campo dos estudos do conhecimento.

No grupo em destaque, desde 2010 os estudos em Grounded Theory vem sendo desenvolvidos de maneira a fortalecer o uso do método como um elemento estratégico para o estudo de fenômenos de alta complexidade, consolidando a indicação de teorias substantivas para a resolução dos fenômenos ou mesmo para o entendimento das relações com constituam as conexões entre os objetos em estudo as visões de mundo em discussão. No bojo de suas características, os três estudos pinçados para a análise são orientados pela mesma docente, neste caso a Professora Doutora Marina Keiko Nakayama, e se apresentam como teses de doutorado.

O trabalho de Pacheco (2010) desenvolveu um estudo sobre a relação entre a permanência e a evasão na educação superior, considerandoos temas emergentes em um contexto altamente complexo, tal como o da educação superior. $O$ uso da Grounded Theory, nesse sentido, abre uma discussão que considera a possibilidade de se constituir um modelo teórico conceitual amparado na gestão do conhecimento. Sob uma ótica paradigmática, o estudo também propõe a caracterização das ações, a partir da visão de Burre e Morgan (1979) relacionadas com objeto de estudo dentro das quatro perspectivas da ciência que são destacadas pelos autores, com ênfase em uma visão interpretativista do fenômeno.

Sob uma ótica interpretativista, o estudo de Rissi (2012) faz um levantamento das relações entre comportamentos específicos e relações tecnologia. $\mathrm{O}$ trabalho tem o mote de compreender as formas pelas quais ocorre o compartilhamento de conhecimento em ambientes virtuais de aprendizagem, utilizando teorias para o suporte do trabalho que congregam a gestão do conhecimento e as teorias de aprendizagem. A Grounded Theory, como estratégia metodológica, é o instrumento que fortalece a construção das teorias e incentiva o uso de uma série de princípios e procedimentos metodológicos que sustentam o desenvolvimento das relações de confiança e do compartilhamento de conhecimento, que são duas das palavras-chave do trabalho.

Já o trabalho de Soares (2012), é explicito em destacar o desenvolvimento de uma teoria substantiva, que segundo Strauss e Corbin (2008) é o principal mote da Grounded Theory. A autora busca identificar, na perspectiva da gestão do conhecimento, as formas pelas quais se constitui uma teoria substantiva (aplicada a um determinado contexto) que possa orientar as situações de conflito em um ambiente de educação a distância. Com o auxílio do estudo de caso, o trabalho destaca que a condição da Grounded Theory como estratégia metodológica permite o uso de diversos procedimentos de pesquisa, permitindo o desenvolvimento de uma teoria estruturada em um conjunto consistente de procedimentos.

Em linhas gerais, em todos os estudos percebemse as seguintes características:

- Todos os estudos abordam fenômenos complexos, cujas produções empíricas são escassas e apenas tangenciam a área de conhecimento em investigação;

-Todos os estudos, em nível de doutorado, buscam a aplicação da Grounded Theory como elemento metodológico e como estratégia de pesquisa, escolhendo campos de conhecimento que são pouco explorados na literatura e que tem um potencial de promover o desenvolvimento de novos estudos;

- Todos os estudos são desenvolvidos sob um paradigma interpretativista, ou pelo menos se posicionam com ênfase nesse contexto. Um deles tem uma abordagem multiparadigmática, mas nas conclusões demonstra que o uso de Grounded Theory observou o viés qualitativo da investigação.

Sob esse prisma, ainda é valido destacar que outros cinco estudos encontram-se em desenvolvimento em nível de doutorado, com a intenção de fortalecer ainda mais o uso da Grounded Theory no campo dos estudos em gestão do conhecimento.

A utilização da grounded theory nos estudos em EGC

Pela análise dos trabalhos, é possível identifica algumas tendências que se estabelecem no programa de Pós-Graduação em Engenharia e Gestão do Conhecimento, considerando o uso da Grounded Theory como estratégia metodológica de pesquisa. Embora sejam apenas três trabalhos que utilizaram o instrumento como instrumento norteador das investigações, já é possível apontar uma tendência para o fortalecimento deste uso em um contexto multidisciplinar, gerando insumos para o desenvolvimento de novos trabalhos. Entre os aspectos que se destacam na criação dessa tendência, encontram-se o aumento das publicações do grupo, o fortalecimento da Grounded Theory no contexto do programa e, sobretudo, o reconhecimento do viés qualitativo e interpretativista em um cenário altamente diversificado, em se tratando de visões epistemológicas distintas.

Com o desenvolvimento dos três trabalhos já defendidos no âmbito do programa e que estão alocados no NEOGAP, outros se encontram em desenvolvimento e influenciaram a produção dos outros que se encontra em fase de qualificação. Desde 2010, são mais de 15 pesquisadores que estão envolvidos com o estudo dessas estratégias metodológicas, defendo a Grounded Theory como elemento metodológico de pesquisa que fortalece os estudos multidisciplinares que utilizam o viés interpratativista e qualitativo como base epistemológica para o desenvolvimento do conhecimento.

Com relação às publicações do grupo, desde o início dos estudos que utilizaram a Grounded Theory, surgem diversos trabalhos que visam o sensibilizar a comunidade multidisciplinar sobre a importância do uso desta consistente estratégia metodológica. Isso se confirma na análise dos estudos de López (2015) e nos estudos de Francisco et. al. (2015), oriundos de uma das contribuições deste grupo de pesquisa e influenciados pelas teses já desenvolvidas, onde possível perceber que a Grounded Theory como estratégia metodológica amplia as possibilidades da construção de teorias substantivas no plano qualitativo da produção do conhecimento. Os trabalhos ainda fortalecem a importância de se compreender a estratégia para estabelecer critérios coerentes de avaliação das pesquisas desenvolvidas nesse contexto, já que estes critérios devem considerar, entre outros aspectos, o contexto em estudo e, sobretudo, a complexidade dos objetos em estudo em consonância com os objetivos da pesquisa.

Com relação ao fortalecimento dos estudos qualitativos em um contexto multidisciplinar, é possível perceber que surgem novos estudos que estão em fase de desenvolvimento e, no caso de teses, em processo de qualificação. Estes estudos se alinham a diversas áreas de conhecimento e convergem com os princípios multidisciplinares no momento em que abordam as áreas da tecnologia da informação, da educação à distância e, nos casos mais recentes, da gestão universitária. Isso mostra a tendência que se criou no programa para o uso da Grounded Theory em uma determinada linha de pesquisa, influenciando outros estudos em investigação qualitativa que devem surgir nos próximos anos.

Em relação às características, é possível identificar que todos os estudos em desenvolvimento são relacionados com a produção de teorias substantivas, que elevam os objetivos em estudo à condição de processo social básico, permitindo que a complexidade destes objetos possa ser compreendida em sua essência, fortalecendo ainda mais a visão de mundo do indivíduo na interpretação 
de um determinado contexto. As teorias substantivas criadas por estes trabalhos, portanto, se consolidam com as principais contribuições destes materiais para a comunidade científica.

Por fim, os estudos desenvolvidos utilizando a Grounded Theory como estratégia metodologia, influenciam outros estudos que se posicionam sob o viés qualitativo e interpretativista, além de contribuir para o uso de procedimentos alinhados com o plano qualitativo de pesquisa. A entrevista em profundidade, as narrativas e a triangulação, como elementos da pesquisa qualitativa, tal como é identificado no trabalho de Quevedo (2013) e Lapolli (2014), entre outros estudos.

\section{CONSIDERAÇÕES FINAIS}

Assim a teoria desenvolvida por Glaser e Staus (1967), apresenta como característica do método, um processo contínuo e sistemático de coleta e análise para geração e verificação dos resultados. Os dados coletados revelam o comportamento dos indivíduos em face de situações específicas. São reconstruções da experiência. A coleta por meio de múltiplas fontes é aconselhada, mas a análise textual das transcrições das entrevistas é mais frequente. Cabe ao pesquisador, em conjunto com os sujeitos, recontar e explicar essas experiências por meio de uma teoria: um conjunto integrado de proposições que explicam a variação da ocorrência de um fenômeno social. A diferença é que ao contrário das metodologias tradicionais a teoria gerada pela Grounded Theory é substantiva. Uma das diferenças entre a teoria formal e a teoria substantiva é que, enquanto a teoria formal positivista é mais gera e aplica-se a um espectro maior de disciplinas e problemas, a segunda é específica. Explica uma "realidade", tornada real pelos sujeitos, e não uma verdade absoluta desprovida de valor. (UHLMANN \& ERDMANN, 2014)

Bandeira-de-Mello e Cunha (2006) acentuam que o método da Grounded Theory pode se amplamente utilizado nos estudos da área da Administração, devido ao estudo da interrelação entre os sujeitos para compreender os fenômeno

organizacionais. Os autores chamam a atenção para o escopo restrito deste método, que cons em gerar uma teoria específica para determinado grupo ou situação e não visa generalizar além da sua área substantiva. Em uma pesquisa que pretende gerar uma teoria substantiva nos moldes da Grounded Theory, espera-se que seja demonstrada lacuna das teorias existentes. Em outras palavras, a literatura insuficiente para explicar determinado fenômeno condiciona o uso da grounded theory. A motivação dos idealizadores ao desenvolver a Grounded Theory partiu da decisão de fazer frente à dominação dos métodos hipotético-dedutivos da Sociologia da época e oferecer uma estratégia rigorosa de desenvolvimento de teoria a partir dos dados. Ambos defendiam que as teorias existentes eram muito abstratas, ou pouco desenvolvidas para serem testadas (WELLS, 1995).

Destarte em um contexto multidisciplinar é notável o aumento da Grounded Theory como metodologia de pesquisa dos trabalhos do Programa de Pós Graduação de Engenharia e Gestão do Conhecimento. Essa tendência contextualiza e acaba por validar a eficácia do método criado pelos sociólogos Glaser e Strauss (1967), que usa a analise qualitativa de dados para acrescentar ou trazer novos conhecimentos ao objeto de estudo. É pertinente ressaltar que a Grounded Theory tem como principal objetivo a identificação, desenvolvimento e o relacionamento de conceitos não possuindo o foco de prova, mas sim do somatório de novas perspectivas ao entendimento do fenomeno em estudo.

BANDEIRA-DE-MELLO, R; CUNHA, C. J C A Grounded Theory. In: GODOI, C. K.; BANDEIRADEMELlO, R.; SILVA, A. B. (Org.). Pesquisa em Estudos Organizacionais: paradigmas, Estratégias e Métodos. São Paulo: Saraiva, 2006.

BURRELL, G.; MORGAN, G. Sociological
Paradigms and Organizational Analysis: Elements of the Sociology of Corporate Life. London: Heinemann, 1979.

BRUUN, H.; HUKKINEN, J.; HUUTONIEMI, K.; KLEIN, J. T. Promoting interdisciplinar research: the case of the academy of Finland. Academy of Finland. 2005

CAPES. Plano nacional de Pós-Graduação 20112020. Coordenação de Pessoal de Nível Superior. Brasília 2010. v.1.

CHARMAZ, Kathy Constructing grounded theory: a practical guide through qualitative research. London: SagePublications, 2006

COMTE, A. Curso de filosofia positiva: discurso sobre o espírito positivo; Discurso preliminar sobre o conjunto do positivismo; Catecismo positivista São Paulo : Abril Cultural, 1978

CRESWELL, J. W; PLANO CLARK, V. L. Pesquisa de métodos mistos. Métodos de Pesquisa. 2.ed. Porto Alegre: Penso. 2013.

DEY, I. Grounding grounded theory: guidelines for qualitative inquiry. San Diego: Academic Press, 1999.

FAVA, R. Educação 3.0: aplicando o PDCA nas instituições de ensino, São Paulo: Saraiva. 2014.

FRANCISCO, T. H.A.; NAKAYAMA, M. K.; OLIVEIRA, P.C.; SCHMIDT, C. N. SOUZA, I. R.. O Uso da Grounded Theory no campo da Administração Universitária: possibilidades e desafios em um campo científico emergente.

CONGRESSO IBERO-AMERICANO EM INVESTIGAC̃̃O QUALITATIVA.,4. Anais. UFG, Aracaju, 2015.
GLASER, B., G.; STRAUSS, A., L. The Discovery of Grounded Theory: strategies for qualitative research. New York: Aldine de Gruyter. 1967.

GODOY, A. S. Pesquisa qualitativa: tipos fundamentais. Revista de Administração de Empresas, São Paulo, v. 35, n. 3, p. 20-29, mai/ jun, 1995.

LAPOLLI, M.. Visualização do conhecimento por meio de narrativas infográficas na web voltadas para surdos em comunidades de prática. 279f. Tese (Doutorado) -Programa de Pós-Graduação em Engenharia e Gestão do Conhecimento. Universidade Federal de Santa Catarina, Centro Tecnológico. Florianópolis, SC, 2014.

LOCKE, K. Grounded theory in management research. London: Sage, 2001.

LOPEZ, M. K.; OLIVEIRA, P. C.; NUNES, C. S.; AKAYAMA, M. K Análise da utilizaça da Grounded Theory (Teoria Fundamentada em Dados) na produção científica brasileira entre 2008 e 2012. RBPG, Brasília, v. 11, n. 24, p.485 - 510, junho de 2014.

MAANEN, J. V. Reclaiming qualitaive methods for organizacional research: a preface. In Administrative Science Quarterly, v.24, n. 4, Dec. 1979.

MILLS, J.; BONNER, A.; FRANCIS, K.. The development of constructivist Grounded Theory. International Journal of Qualitative Methods, v.5, n.1, Mar. 2006.

MOREIRA, D. A.. O método fenomenológico na pesquisa. São Paulo: Pioneira Thomson, 2002. PACHECO, Andressa Sasaki Vasques. Evasão permanência dos estudantes de um curso de administracão do sistema Universidade Aberta do Brasil: uma teoria fundamentada em fatos 
e na gestão do conhecimento. Tese. Programa Catarina, Florianópolis, 2012.

de Pós-Graduacão em Engenharia e Gestão do

Conhecimento. Universidade Federal de Santa

Catarina, Centro Tecnológico. Florianópolis, SC, 2010.

PINTO, M. R.; SANTOS, L. L. S. The Grounded

Theory as methodological approach: reports of a field experience. Revista: Organizações e Sociedade, Salvador, v.19, n. 62, July/Sept. 2012

QUEVEDO, S. R. P. de. Narrativas hipermidiáticas para ambiente virtual de aprendizagem inclusivo. 380f. Tese (Doutorado) Programa de Pós-Graduação em Engenharia e Gestão do Conhecimento Universidade Federal de Santa Catarina, Centro Tecnológico. Florianópolis, SC, 2013.

RISSI, M. A confiança e as relações interpessoais assegurando o compartilhamento do conhecimento no ambiente virtual de aprendizagem. 2013. $204 \mathrm{f}$. Tese. (Doutorado em Engenharia e Gestão do Conhecimento) - Programa de Pós-Graduação em Engenharia e Gestão do Conhecimento, Universidade Federal de Santa Catarina, Florianópolis, 2012

ROSSO, S. D; BANDEIRA, L., COSTA, A. Pluralidade e Diversidade das Ciências Sociais: uma contribuição para a epistemologia da ciência. Sociedade e Estado, Brasília, v. 17, n. 2, p. 231246, jul./dez. 2002.

SILVEIRA, F. L.. A metodologia dos programas de pesquisa: a epistemologia de Imre Lakatos. Revista de Enseñanza de la Física, Cordoba, v. 10, n. 2: 56-63, 1997.

SOARES, A. P. Gestão do conhecimento e conflitos interorganizacionais em EaD: construção de uma teoria substantiva. $470 \mathrm{f}$. Tese (Doutorado em Engenharia e Gestão do Conhecimento) - Programa de Pós-Graduação em Engenharia e Gestão do Conhecimento, Universidade Federal de Santa
S O M M E R M A N, A. I n t e r o u transdisciplinaridade? da fragmentação disciplinar ao novo diálogo entre os saberes. São Paulo: Paulus. Coleção questões fundamentais da educação. 2006.

STRAUSS, A.; CORBIN, J. Basics of qualitative research: techniques and procedures for developing Grounded Theory. 2 nd. Thounsand Oaks: Sage Publications, 1998.

STRAUSS A.; CORBIN, J. Basics of Qualitative Research: grounded theory procedures and techniques. London: Sage Publications, 1990.

STRAUSS, A.; CORBIN, J. Pesquisa qualitativa: técnicas e procedimentos para o desenvolvimento de uma teoria fundamentada. 2.ed. Porto Alegre: Artmed, 2008.

TAYLOR, S. J.; BOGDAN, R. Introduction to qualitative research methods: the search for meanings. John Wiley \& Sons. 2. ed. 1984.

UHLMANN, V. O., ERDMANN, R. H. Usos e aplicações da grounded theory em administração. SIMPOI, 2014.

WELLS, K. The strategy of grounded theory: possibilities and problems. Social Work Research. v. 19, n. $1,1995$.

WHITTEMORE, Robin; KNAFL, Kathleen. The integrative review: updated methodology. Journal of Advanced Nursing, v. 52 , n.5, p. 546-553, 2005.
MUSETTI, Tiago Fernando*. - Universidade Federal de São Carlos - Departamento de Engenharia de Produção, Mestrado: Engenharia de Produção (SEP-EESC/USP) - 2013 a 2015, Doutorando: Departamento de Engenharia de Produção (DEP/UFSCar) ; ESCRIVÃO FILHO, Edmundo. - Universidade de São Paulo, Escola de Engenharia de São Carlos, Departamento de Engenharia de Produção (SEP-EESC/ USP), Doutor em Engenharia de Produção (1995) e Professor Associado (2006; ALVES FILHO, Alceu Gomes. - Departamento de Engenharia de Produção (SEP-EESC/USP), Doutor em Engenharia de Produção (1991) e Professor Associado (2006); PERUSSI FILHO, Sérgio. - Professor Doutor, Centro Universitário Central Paulista - Departamento de Engenharia de Produção (UNICEP), Doutor em Engenharia de Produção (2006).

*Autor para correspondência e-mail: tiagoferm@yahoo.com.br

Recebido em: 09/08/2017
Aprovacão final em: $15 / 122017$

Resumo

Este artigo tem o objetivo de analisar o uso das fontes de informações do ambiente organizacional em pequenas empresas. A abordagem de pesquisa utilizada foi a qualitativa, por meio de estudo de casos múltiplos, aplicados em pequenas empresas, nos setores metal mecânico e de base tecnológica. Como resultado, descobriu-se que as principais fontes externas de informações são os clientes, o departamento comercial e os empresários. A principal contribuição desta pesquisa é mostrar que não há diferenças significantes nas fontes de informação que os empresários, de ambos os setores, utilizam para obter informações sobre $\mathrm{o}$ ambiente organizacional.

Palavras-chave: Pequena Empresa; Ambiente Organizacional; Fontes de Informações sobre o Ambiente Organizacional; Monitoramento do Ambiente Organizacional.

INFORMATION SOURCES OF THE ORGANIZATIONAL ENVIRONMENT IN SMALL BUSINESSES

Abstract

This article aims to analyze the use of organizational environment information sources in small businesses. The method used in this research was the qualitative approach, through multiple case studies, applied in metal mechanic and technological based small businesses. As a result, the main external information sources are costumers, commercial department and entrepreneurs. The main contribution of this research is that there are no significant differences in the sources, which are used by both sectors to obtain information on organizational environment.

KEYwORDS: Small Business; Organizational Environment; Organizational Environment Information Sources; Organizational Environment Monitoring. 